\title{
Habilidades socioemocionales en universitarios que aspiran emprender
}

\author{
Martha Leticia Gaeta González ${ }^{1}$ \\ Luis Gerardo Ortíz Lack²
}

Recibido 27 de nov $2018 \cdot$ Aceptado 2 de mayo 2019

\begin{abstract}
RESUMEN
El proceso de emprendimiento supone un reto para los universitarios, al enfrentarlos a un contexto social dinámico, que exige estrategias para favorecer una idea de negocio rentable y perfiles profesionales que permitan afrontar los desafíos personales y del entorno durante el desarrollo del emprendimiento. Las habilidades socioemocionales se consideran herramientas fundamentales para el emprendimiento, al permitir a los estudiantes gestionar sus recursos personales con apoyo del contexto, para el desarrollo de estrategias y comportamientos de incidencia en el entorno. En el contexto universitario mexicano, este trabajo se realizó con el propósito de identificar, mediante la aplicación de un cuestionario estandarizado, las habilidades socioemocionales de los estudiantes vinculadas a la aspiración de emprender. Los resultados muestran un nivel medio en las habilidades socioemocionales de los universitarios que aspiran emprender. Se enfatiza la importancia de considerar la dimensión afectiva en los planes de estudio enfocados al emprendimiento universitario.
\end{abstract}

Palabras clave: autorregulación, emprendedores, educación emocional, habilidades.

\section{Socioemotional Skills in University Students Who Want to Start Their Own Business}

\section{ABSTRACT}

The entrepreneurship process is a challenge for university students, as it puts them face to face with a dynamic social context that demands strategies to support a profitable business idea and professional profiles in order to confront personal and external challenges during the development of the business start-up process. Socioemotional skills are considered fundamental tools for entrepreneurship, by allowing students to manage their personal resources with the support of their surroundings, in order to develop strategies and behaviors to affect their surroundings. In the Mexican university context, this research study applied a standardized questionnaire for the purpose of identifying socioemotional skills in students who want to start their own business. The results show an intermediate level in terms of the socioemotional skills of university students looking to start their own business, and stress

Profesora investigadora, Decanato de Artes y Humanidades, Universidad Popular Autónoma del Estado de Puebla. Contacto: marthaleticia.gaeta@upaep.mx

2 Egresado de Maestría, Decanato de Artes y Humanidades, Universidad Popular Autónoma del Estado de Puebla. Contacto: luislackII@gmail.com 
the importance of considering the affective dimension in curricula focused on university student entrepreneurship.

Key words: self-regulations, entrepreneurs, emotional education, skills.

\section{Introducción}

Los emprendedores son personas que identifican nuevas oportunidades socioeconómicas que aún no han sido satisfechas, al proponer un plan de crecimiento enfocado a una población con necesidades específicas, favoreciendo así el contexto social en el que se desarrollan. En México, las reformas económicas han buscado promover la innovación e impulsar planes de negocio que impacten a los diversos contextos socioeconómicos del país. El desarrollo de PyMES ha sido la propuesta para impulsar el crecimiento social y monetario del país.

Así, durante 2014 se consolidó una gran cantidad de empresas dentro de este esquema, llegando a ser el $94 \%$ al cierre del censo ecónómico del Instituto Nacional de Estadística y Geografía (La Jornada, 2015). A partir de ello, el gobierno de la república ha desarrollado el Instituto Nacional del Emprendedor (INADEM, 2013), organización mediante la cual es posible el desarrollo de modelos de negocio estables y con una estructura económicamente viable a corto, mediano y largo plazo. Lo anterior se ha llevado a cabo gracias a capacitaciones y diversos incentivos a los emprendedores, con el fin de obtener recursos del contexto y acceder a espacios de crecimiento para su plan de negocios.

En este contexto, el Global Entrepreneurship Monitor (Instituto Tecnológico de Estudios Superiores de Monterrey, 2015) señala a 2014 como un año importante en el ámbito del emprendimiento, al indicar el alza de los índices por parte del mercado mexicano, proyectando un panorama económico favorecedor en este sector. Dentro del ámbito empresarial, específicamente el emprendimiento, el desarrollo de talento para este tipo de perfiles es de vital importancia, pues gracias a ello es posible desarrollar nuevas propuestas de negocio que permitan a los consumidores satisfacer sus necesidades $y$, al mismo tiempo, generar un derrame económico para el país. 
No obstante lo anterior, el desarrollo de los modelos de negocio aún no logra construir un esquema sostenible para las PyMES; durante 2013 únicamente se logró un aumento de PyMES del $22 \%$ (Santa Rita, 2013) y, posterior a la incubación del negocio, la rentabilidad de los planes de negocios no ha sido la esperada, llegando a concluir actividades el 82,5\% de PyMES (Flores-Kelly, 20I3). Lo anterior denota la necesidad de desarrollar una estructura que posibilite el desarrollo sostenible dentro de la incubación y puesta en marcha de este tipo de negocios. Para ello, la persona que emprende debe adquirir, además de los conocimientos, las habilidades socioemocionales que le permitan resolver las distintas problemáticas y tomar decisiones oportunas en el proceso emprendedor.

En este sentido, los centros educativos - como las universidadesson espacios de convivencia así como de desarrollo de ideas innovadoras en materia empresarial, donde los estudiantes tienen la oportunidad, dentro de las actividades académicas, de crear planes de negocios que atiendan a las necesidades no cubiertas de ciertos sectores de la población y favorecer la empleabilidad del contexto local y nacional.

Dentro del desarrollo curricular, los diversos programas de educación superior enfocados al área de emprendimiento cuentan con elementos en los que se gestionan contenidos enfocados a la incubación de proyectos de emprendimiento a corto, mediano y largo plazo, vinculando a los estudiantes con las necesidades del entorno en el que se desarrollan. Dado ello, es importante identificar si estas propuestas educativas han permeado las dimensiones personales del individuo, con el fin de apoyar integralmente la generación de proyectos de emprendimiento innovadores, que realmente atiendan a las necesidades del proyecto de incubación empresarial, con un énfasis en el desarrollo socioemocional de las personas.

\section{Referentes teóricos}

Un emprendedor es considerado como un individuo con la capacidad de identificar necesidades que aún no han sido cubiertas en un contexto determinado, desarrollando así una idea de negocio para satisfacer las 
carencias del entorno (Ibáñez, 2002). Dado que la rentabilidad de un país depende en gran medida de la iniciativa de sus habitantes y las propuestas económicas desarrolladas, el emprendimiento permite aprovechar de una mejor manera los recursos económicos del territorio nacional, favoreciendo espacios de crecimiento personal, profesional y social de los ciudadanos, así como una administración más balanceada, mediante la innovación en diversos ámbitos como la agricultura, tecnología y la inversión extranjera.

El emprendimiento es un proceso que, a lo largo de la historia, se ha ido modificando. En las primeras etapas de su definición, el emprendedor era visualizado como un individuo que pretendía afrontar riesgos para el desarrollo de nuevas ideas y, posteriormente, producir algún bien o servicio, tomando en cuenta las dimensiones psicológicas únicamente desde una concepción general, en la cual los esquemas cognitivos de pensamiento y los diversos grupos de acciones desarrollados eran la pauta para emprender cierta idea innovadora. Posteriormente se empezó a considerar la influencia del contexto social en el desarrollo del individuo y en la incubación del plan de negocio, siendo este contexto la base sobre la cual el emprendedor puede llegar a favorecer la innovación (Herrera y Montoya, 20I3). Cabe señalar que, en el proceso de emprender, las dimensiones social y emocional del individuo juegan un papel fundamental, pues es a partir de ellas que el individuo desarrolla una interacción con el ambiente para gestionar adecuadamente sus metas del proyecto a corto, mediano y largo plazo.

En tal sentido, los centros educativos se consideran espacios en los que las personas desarrollan sus habilidades para enfrentarse a los retos de la vida laboral, espacios de aprendizaje para acceder a nuevos esquemas de pensamiento. Con base en lo anterior, el contexto educativo universitario es un excelente ámbito para desarrollar planes innovadores, en los se propicie la inserción curricular de contenidos que faciliten experiencias de aprendizaje en los estudiantes enfocadas al emprendimiento, con el fin de estimular la gestión de habilidades sociales, emocionales y de incubación empresarial, fomentando la consecución de proyectos en los que se favorezca el desarrollo económico y social. 
Dichas propuestas deben desarrollarse desde los programas de emprendimiento, que favorezcan el desarrollo de proyectos innovadores con énfasis en el desarrollo humano, adecuando así los planes de estudio vigentes para proponer estructuras curriculares integrales. Lo anterior debido a que en la mayoría de los planes de estudio enfocados al área de emprendimiento se da importancia en gran medida a los factores económicos, financieros y administrativos; sin embargo, las habilidades socioemocionales han sido dejadas de lado en cierto sentido. Estas últimas juegan un papel fundamental en el desarrollo de esquemas para el manejo efectivo de sí mismo y el autoconocimiento cuando se interactúa con problemáticas, facilitando así encontrar mejores soluciones en el desarrollo de procesos para la innovación (Durán \& Arias, 2015). Por lo anterior, las habilidades socioemocionales son importantes para favorecer la aspiración por emprender en los estudiantes.

De esta forma, las aspiraciones constituyen un proceso de carácter psicológico y social en el cual la persona se siente atraído hacia una meta (González \& Valenzuela. 2016). Así, la aspiración por emprender implica el deseo por parte del sujeto de desarrollar una idea de negocio innovadora que atienda una necesidad no cubierta en un contexto determinado. Tomando en cuenta la aspiración por emprender, es importante identificar el rol del contexto en la claridad y regulación emocional del individuo durante el proceso. Saarni (200Ia, 200Ib) explica la importancia del ambiente en el proceso del desarrollo de habilidades socioemocionales adecuadas, al plantear el rol del contexto social como un espacio didáctico en el que los indivduos adquieren un lenguaje social para el desarrollo de sus emociones.

Ello influye en la gestión de comportamientos emocionales que el individuo desea obtener, tanto en sí mismo como en los demás, gracias a la interacción con sus pares y otros elementos importantes del esquema social. El espacio educativo, como hemos señalado, al ser un medio social en el que el estudiante convive en gran medida con diversos actores, puede fortalecer su autoconcepción con miras al emprendimiento, gracias a las experiencias de aprendizaje en un ambiente de colaboración, diálogo y de desarrollo personal y 
social. Se requiere, por tanto, una estructura curricular que atienda las dimensiones emocionales y cognitivas del individuo, tomando como base la interacción que éste tiene con su entorno social, lo que facilita la adquisición de habilidades socioemocionales que permiten la adaptación del sujeto durante su proceso formativo, facilitando así la aspiración por emprender en sus estudiantes.

La formación emprendedora en el contexto educativo, favorece una visión positiva hacia el comportamiento empresarial innovador $y$, por ende, el desarrollo de una visión hacia el emprendimiento (Martínez \& Campos, 20I4). Por ello, la innovación educativa juega un rol fundamental en el desarrollo de estructuras curriculares adecuadas a las necesidades de los estudiantes emprendedores, tomando en cuenta su perfil de aprendizaje a nivel emocional y cognitivo, favoreciendo así el logro de aprendizajes en los se favorezca el desarrollo de ciertas acciones.

La influencia de las propuestas educativas que atienden la dimensión personal así como las competencias administrativas en el desarrollo de una propuesta innovadora de negocio, son por tanto una tarea docente que favorece en los estudiantes nuevos esquemas de autoconocimiento, vinculación de los contenidos aprendidos en el aula con sus actividades diarias, así como estrategias de creatividad para ahondar de una mejor manera las propuestas de innovación emprendedora (Orazem, Jolly \& Yu, 20I5; Kenney, 20I I; Núñez, Mercado \& Madrigal, 20I4). Desarrollar espacios didácticos en los que se promueva la dimensión personal, propiciando un mejor afrontamiento de las circunstancias a las cuales se enfrenta durante el proyecto innovador (Scafarelli \& García, 2010), consideramos, permitirá un mayor desarrollo profesional y de innovación emprendedora que facilite el desarrollo de planes de negocio factibles y, por ende, el crecimiento económico del país.

Es así como el desarrollo de prácticas efectivas y con sentido dentro de la institución educativa permite desarrollar el sentido de cooperación entre pares, la evaluación del proceso de aprendizaje así como la motivación por parte de los estudiantes por medio de contenidos que incidan positivamente en su autoestima (Marchesi, 
2009). De aquí la importancia de generar ambientes educativos que fortalezcan el vínculo entre el estudiante con su entorno, en los que se promueva su aspiración por emprender, siendo el contexto social un facilitador de herramientas para el desarrollo de ciertos comportamientos que permiten acceder a esquemas de pensamiento necesarios para desarrollar aprendizajes a corto, mediano y largo plazo (Gaeta \& López, 2013).

Por otro lado, la concepción del individuo acerca de sí mismo, así como de las habilidades que posee, constituyen un factor fundamental en el desarrollo de su aspiración por emprender. Por medio de estas concepciones, la persona puede llegar a generar estructuras de pensamiento relevantes para sí, con el fin de construir esquemas cognitivos que organicen la información aprendida anteriormente y proyectarla en el momento adecuado para el logro de metas que se plantee (Kassin, Fein \& Markus, 20I0). La visión generada por parte del sujeto hacia sí mismo favorece el interés por lograr cierta actividad, al identificar que cuenta con las herramientas necesarias para proyectar una respuesta adecuada; lo anterior se logra gracias al establecimiento de objetivos que le permiten plantearse metas para desarrollar una experiencia favorable (Blanco, 2009). En este sentido, las habilidades socioemocionales tienen un rol fundamental para el desarrollo de del autoconcepto y viceversa.

La concepción del individuo junto con sus habilidades socioemocionales se ven favorecidas por el apoyo del contexto social donde se desarrolla, al lograr vincular las experiencias aprendidas en conjunto con los elementos emocionales. De esta forma, las habilidades socioemocionales se pueden fortalecer gracias al acompañamiento de agentes del contexto social en el que se sitúa el estudiante, evaluando de una mejor manera las situaciones a las que se enfrenta día a día, para así lograr un mejor abordaje emocional de la problemática presentada (Limonero, Tomás, Fernández, Gómez \& Ardilla, 2012). De aquí la importancia del contexto social, en este caso de la institución educativa donde se forman los universitarios y de los procesos formativos que ahí se desarrollan, para favorecer sus habilidades socioemocionales en su aspiración por emprender. 
A partir de los referentes anteriores, el objetivo de la investigación es identificar las habilidades socioemocionales vinculadas a la aspiración por emprender en los universitarios.

\section{Método}

El método de este estudio es cuantitativo, con un diseño de encuesta, a partir de la aplicación de un instrumento estandarizado (Casas, Repullo \& Donado, 2003).

\subsection{Participantes}

Estudiantes de una universidad privada en el estado de Puebla, México, que se encontraban en el programa curricular de emprendedores. El muestreo fue no probabilístico, con una muestra de $n=58(79 \%$ de la población total, $N=73)$ teniendo como criterios de inclusión alumnos inscritos en el periodo otoño 2017 en la asignatura de Emprendimiento II, con enfoque a la incubación de su propia empresa.

\subsection{Instrumentos}

Las variables que se midieron fueron la aspiración por emprender y las habilidades socioemocionales de los universitarios. Para ello se utilizaron dos de las dimensiones del Cuestionario de Adaptación Socioemocional en Escolares, el cual es un instrumento estandarizado previamente validado (Mathinsen, Merino, Castro, Mora y Navarro, 20I I). Estas dimensiones son: (I) habilidades emocionales, con 10 ítems (ej. Tengo dificultad de expresar lo que siento) y (2) habilidades sociales, con 6 ítems (ej. Mis compañeros me consideran como una persona a la que es importante invitar). Los ítems del instrumento se revisaron y se ajustó su redacción al contexto universitario, a partir de su aplicación a un grupo piloto de cinco estudiantes. Asimismo, se incluyó una pregunta al inicio del cuestionario acerca de si aspiraban emprender. El instrumento total cuenta con un alfa de Cronbach de .936, mostrando un índice de confiabilidad adecuado (tabla I). 
Tabla I. Confiabilidad del instrumento

\begin{tabular}{|l|c|c|}
\hline \multicolumn{1}{|c|}{ FACTOR } & ITEMS & $\alpha$ \\
\hline Habilidades emocionales & I0 & .735 \\
\hline Habilidades sociales & 6 & .686 \\
\hline Total & II2 & .936 \\
\hline
\end{tabular}

Fuente: elaboración propia, 2019.

\subsection{Procedimiento}

Se solicitó autorización para el desarrollo del estudio a la coordinación del programa de emprendedores de la universidad y se invitó a los estudiantes conformantes de la muestra para su participación voluntaria. Posteriormente se procedió al análisis estadístico para el tratamiento de los datos, mediante el programa estadístico SPSS, versión 22 .

\section{Resultados}

La media de edad de los participantes fue de 23 años (DT=I.I8). De los 58 estudiantes participantes el sexo femenino fue el predominante (54\%). Durante el análisis se encontró que no existen diferencias por edad en las habilidades socioemocionales.

Las licenciaturas cursadas por los estudiantes de la muestra se pueden visualizar en la tabla 2. Las licenciaturas con mayor número de estudiantes inmersos en la modalidad emprendedora son Diseño gráfico y digital / Diseño y producción publicitaria (I4\%), seguido por el área de Mercadotécnica (I2\%), Odontología (I0\%) y, en menor grado, Administración de empresas/ Cine y producción audiovisual (7\%), Administración de instituciones/Inteligencia de negocios (5\%), Arquitectura/Contaduría pública y alta dirección/ Educación/Médico Veterinario Zootecnista (3\%), y con el menor número Gastronomía/Idiomas/Ingeniería civil/Ingeniería industrial/Mecatrónica/Nutrición y Pedagogía (2\%). 
Tabla 2. Estudiantes que aspiran a emprender por área profesional

\begin{tabular}{|l|c|c|}
\hline \multicolumn{1}{|c|}{ LICENCIATURA } & $\begin{array}{c}\text { NÚMERO DE } \\
\text { PARTICIPANTES }\end{array}$ & $\%$ \\
\hline Administración de empresas & 4 & $7 \%$ \\
\hline Administración de instituciones & 3 & $5 \%$ \\
\hline Arquitectura & 2 & $3 \%$ \\
\hline Cine y producción audiovisual & 4 & $7 \%$ \\
\hline Contaduría pública y alta dirección & 2 & $3 \%$ \\
\hline Diseño gráfico y digital & 8 & $14 \%$ \\
\hline Diseño y producción publicitaria & 8 & $14 \%$ \\
\hline Educación & 2 & $3 \%$ \\
\hline Gastronomía & $\mathrm{I}$ & $2 \%$ \\
\hline Idiomas & $\mathrm{I}$ & $2 \%$ \\
\hline Ingeniería civil & $\mathrm{I}$ & $2 \%$ \\
\hline Ingeniería industrial & $\mathrm{I}$ & $2 \%$ \\
\hline Inteligencia de negocios & 3 & $5 \%$ \\
\hline Mecatrónica & $\mathrm{I}$ & $2 \%$ \\
\hline Mercadotecnia & 7 & $\mathrm{I} \%$ \\
\hline Médico Veterinario Zootecnista & 2 & $3 \%$ \\
\hline Nutrición & $\mathrm{I}$ & $2 \%$ \\
\hline Odontología. & 6 & $10 \%$ \\
\hline Pedagogía. & $\mathrm{I}$ & $2 \%$ \\
\hline Total alumnos & 58 & $100 \%$ \\
\hline
\end{tabular}

Fuente: elaboración propia, 2019.

En cuanto a la aspiración por emprender, el área profesional de administración de comercios y servicios profesionales es donde se cuenta con un mayor porcentaje de estudiantes que aspiran emprender (62\%), y el área de la salud la que menos cuenta con estudiantes con esta aspiración ( $9 \%$ ) (tabla 3 ). 
Tabla 3. Aspiración por emprender por áreas profesionales

\begin{tabular}{|l|c|c|}
\hline \multicolumn{1}{|c|}{ ÁREA PROFESIONAL } & $\begin{array}{c}\text { NÚMERO DE } \\
\text { ESTUDIANTES }\end{array}$ & $\%$ \\
\hline $\begin{array}{l}\text { Industria de alimentos y } \\
\text { restaurantes. }\end{array}$ & 9 & $16 \%$ \\
\hline Área de la salud. & 5 & $9 \%$ \\
\hline $\begin{array}{l}\text { Administración de comercios y } \\
\text { servicios profesionales. }\end{array}$ & 36 & $62 \%$ \\
\hline No respondió. & 7 & $12 \%$ \\
\hline No sabe. & $\mathrm{I}$ & $2 \%$ \\
\hline Total & 58 & $100 \%$ \\
\hline
\end{tabular}

Fuente: elaboración propia, 2019.

Respecto del desarrollo de habilidades socioemocionales, el $68 \%$ $(n=38)$ de los participantes se encuentran en un nivel medio, mientras que el $32 \%(n=I 8)$ se encuentra en un nivel alto (tabla 4$)$.

Tabla 4. Nivel de desarrollo de habilidades socioemocionales de los estudiantes

\begin{tabular}{|l|c|c|}
\hline Nivel & Percentil del instrumento & Porcentaje de sujetos \\
\hline Medio & 53.3 & 38 sujetos $(66 \%)$ \\
\hline Alto & 80 & 18 sujetos $(31 \%)$ \\
\hline
\end{tabular}

Fuente: elaboración propia, 2019.

De manera más específica, en la tabla 5 se describen los aspectos que mostraron una mayor frecuencia de respuesta para el desarrollo de habilidades socioemocionales. Sentirse capaz de comunicarse entre pares y relacionarse con personas adultas fueron los aspectos más mencionados. Además, considerarse como una persona alegre y sentirse feliz se referenciaron como aspectos preponderantes por parte de los participantes (tabla 5). 
Tabla 5. Desarrollo de habilidades socioemocionales por ítem

\begin{tabular}{|l|c|c|c|c|c|c|}
\hline \multirow{2}{*}{$\begin{array}{l}\text { Ítems con } \\
\text { mayor } \\
\text { frecuencia de } \\
\text { respuesta }\end{array}$} & Frecuencia & I & 2 & 3 & 4 & 5 \\
\cline { 3 - 7 } & & & & & & \\
\hline $\begin{array}{l}\text { P62. Siento } \\
\text { que soy } \\
\text { capaz de } \\
\text { relacionarme } \\
\text { con adultos }\end{array}$ & 32 & $7.14 \%$ & $0 \%$ & $9.61 \%$ & $26.78 \%$ & $57.14 \%$ \\
\hline $\begin{array}{l}\text { P70. Suelo } \\
\text { sentirme feliz }\end{array}$ & 32 & $1.78 \%$ & $1.78 \%$ & $8.92 \%$ & $30.35 \%$ & $57.14 \%$ \\
\hline $\begin{array}{l}\text { P73. Me gusta } \\
\text { participar en } \\
\text { actividades } \\
\text { como fiestas } \\
\text { y reuniones } \\
\text { de amigos y } \\
\text { compañeros }\end{array}$ & 30 & $0 \%$ & $10.71 \%$ & $8.92 \%$ & $26.78 \%$ & $53.57 \%$ \\
\hline $\begin{array}{l}\text { P74. Me } \\
\text { considero una } \\
\text { persona alegre }\end{array}$ & 35 & $0 \%$ & $1.78 \%$ & $16.07 \%$ & $19.64 \%$ & $62.5 \%$ \\
\hline $\begin{array}{l}\text { P75. No siento } \\
\text { vergüenza } \\
\text { cuando tengo } \\
\text { que hablar con } \\
\text { gente de mi } \\
\text { edad }\end{array}$ & 29 & 0 & $1.78 \%$ & $12.5 \%$ & $33.92 \%$ & $51.78 \%$ \\
\hline
\end{tabular}

Fuente: elaboración propia, 2019.

Se realizó la prueba estadística Mann-Whithney, con el fin de hacer una comparación de habilidades socioemocionales por sexo. No se encontraron diferencias significativas entre hombres y mujeres.

\section{Discusión y conclusiones}

Con base en los resultados, se observa que existe una aspiración de emprender por parte de los universitarios encuestados, mayoritariamente en el área de administración de comercios y servicios profesionales y, en específico, en las licenciaturas de Diseño gráfico y digital, Diseño de producción publicitaria y Mercadotecnia. Por la naturaleza de esta área formativa, es posible inferir que la concentración del mayor 
número de universitarios con la aspiración por emprender se debe a la flexibilidad de integrar diversos aspectos de conocimiento en las ideas de negocio dentro del trayecto formativo de manera curricular. Sin embargo, sería conveniente indagar cuáles son los factores que inciden en esta y en las demás áreas disciplinares para una mayor aspiración (o no) de emprender, con el fin de seguir habilitando nuevos espacios de diálogo y desarrollo, en los que las dimensiones menos favorecidas se puedan atender plenamente, llevando a los alumnos a un estado de motivación por generar nuevos proyectos innovadores.

Respecto del desarrollo de habilidades socioemocionales, los resultados muestran que la mayoría de los estudiantes cuentan con un nivel medio de desarrollo (66\%), y un menor porcentaje cuenta con un nivel alto (3I\%). Como comenta Saarni (200Ia), las competencias socioemocionales favorecen la demostración de emociones durante el desarrollo de cierto comportamiento, apoyando así la realización de ciertas metas planteadas. Las habilidades socioemocionales favorecen la actitud de emprender, al facilitar en el emprendedor las estrategias necesarias para hacer frente a los retos propios del proyecto y propiciar mejores estados de ánimo durante la resolución de problemas (Sánchez \& ArayaVargas, 2013).

Este punto es importante, pues a pesar de existir una aspiración por emprender, no contar con niveles altos de habilidades socioemocionales dentro de un contexto social determinado puede llegar a afectar el desempeño del sujeto durante la incubación de su negocio, arriesgando decaer paulatinamente durante su puesta en marcha. Lo anterior puede desarrollarse a partir de la propuesta curricular, identificando los aspectos clave que puedan incidir desde la formación, para ayudar a los estudiantes a potenciar este tipo de habilidades y así lograr una incubación del proyecto en la que se atienda al ser humano como un ser integral.

La estructura curricular de la línea de emprendimiento puede favorecer este tipo de aspectos, al definir en sus planes de estudio contenidos y estrategias enfocadas al desarrollo de habilidades socioemocionales, en las que el estudiante identifique la importancia 
de la vinculación con el entorno para el pleno desarrollo de su dimensión emocional, la cual es clave en el desarrollo de proyectos con miras al emprendimiento. Este tipo de apoyo por parte de la institución puede llegar a favorecer los resultados en materia de ideas de negocios estruturadas y viables para el contexto al cual se encuentran enfocadas, gracias a la formación del universitario emprendedor con miras al desarrollo de nuevos esquemas para la movilización económica y social de su entorno.

En este sentido, la universidad, como uno de los principales espacios en el proceso de emprendimiento, juega un rol fundamental para la incubación de la idea de negocio, al ampliar las oportunidades del individuo con miras a la generación de proyectos innovadores así como la posible vinculación con otros emprendedores universitarios, facilitando el desarrollo académico dentro de los programas curriculares, en los que se promueva la apertura de esquemas de trabajo enfocados hacia el emprendimiento (Barba-Sanchez \& Atienza-Sahuquillo, 20I I). Favorecer espacios educativos en los que el estudiante cuente con la oportunidad de desarrollar sus habilidades socioemocionales, facilitará su adaptación personal ante los retos y problemáticas formativas y de cara al emprendimiento (Scafarelli y García, 2010; Osorio y Pereira, 201 I).

En suma, tomar en cuenta las habilidades socioemocionales en la formación de los emprendedores en las instituciones de educación superior, desde una visión integral, consideramos, permitirá generar una estrutura de crecimiento innovadora en la que se atienda a la persona como un ser holístico, facilitando los posibles enfrentamientos y decaídas emocionales que forman parte de cualquier poroceso de emprendimiento. Lo anterior ayudará al individuo visualizar el proceso como un reto alcanzable, con el apoyo de su contexto social, para así favorecer una incubación de negocio de manera más integral y enfocada al perfil de cada estudiante.

\section{Referencias bibliográficas}

Barba-Sanchez, V. y Atienza-Sahuquillo, C. (20I I). Reasons to create a new venture: A determinant of entrepreneurial profiles. African jorunal of 
business management, 5(28), I I497-I I504. Recuperado de https:/ /www. researchgate.net/publication/2I6896228_Reasons_to_Create_a_ New_Venture_A_Determinant_of_Entrepreneurial_Profiles

Bisquerra, R. (1996). Métodos de investigación educativa: guía práctica. Barcelona: Ceac.

Blanco, A. (Septiembre-Diciembre, 2009). El modelo cognitivo social del desarrollo de la carrera: revisión de más de una década de investigación empírica. Revista de Educación, (350), 423-445. Recuperado de http:// www.revistaeducacion.mec.es/re350/re350_I8.pdf

Casas, J., Repullo, J.R., Donado, J. (2003). La encuesta como técnica de investigación. Elaboración de cuestionarios y tratamiento estadístico de los datos. Atención Primaria, 32(8), 527-538. Recuperado de http:// www.elsevier.es/es-revista-atencion-primaria-27-articulo-la-encuestacomo-tecnica-investigacion--I3047738

Durán, E. y Arias, D. (julio-diciembre, 2015). Intención emprendedora en estudiantes universitarios: integración de factores cognitivos y sociopersonales. Revista Colombiana de Ciencias Sociales, 6(2), 320-340. Recuperado de http://www.funlam.edu.co/revistas/index.php/ RCCS/article/download/I528/pdf_2I

Flores-Kelly, J. (2013). México necesita menos Pymes. México: Forbes. Recuperado de: http://www.forbes.com.mx/mexico-necesita-menos-pymes/

Flores, L. (2013). Reforma bacendaria, riesgo para Pymes. México: CNNExpansión. Recuperado de: http://www.cnnexpansion.com/ economia/2013/II/22/tips-sobre-la-reforma-fiscal

Gaeta, M.L. y López, C. (2013). Competencias emocionales y rendimiento académico en estudiantes universitarios. Revista electrónica de formación del profesorado, 16(2), I2-25. Recuperado de http://www.redalyc.org/ pdf/2170/217029557002.pdf , doi: http://dx.doi.org/I0.6018/ reifop.16.2.181031

González, A. y Valenzuela, J. (2016). Factores motivacionales y construcción de aspiraciones de estudios universitarios. Evidencia desde un contexto regional chileno. Revista de Pedagogía, 37(I00), I I I-I34.

Herrera, C.E. y Montoya, L.A. (Julio-Diciembre, 2013). El emprendedor: una aproximación a su definición y caracterización. Punto de vista, 4(7), 7-30. Recuperado de https:/ / dialnet.unirioja.es/descarga/articulo/4776922.pdf

Ibáñez, M.A. (2002). Actitudes emprendedoras en los estudiantes universitarios: medición y propuestas de actuación. Madrid: Ediciones Mensajero.

Instituto Nacional del Emprendedor. (2013). ¿Qué es INADEM? Recuperado de https://www.inadem.gob.mx/que_es_inadem.html 
Instituto Tecnológico de Estudios Superiores de Monterrey. (2015). Crece el emprendimiento en el mundo, revela el estudio GEM presentado en el Tec de Monterrey. Recuperado de http://www.itesm.mx/wps/wcm/connect/snc/ portal+informativo/por+tema/negocios/tec_presentaciongem 2014

Kassin, S., Fein, S. y Markus, H.R. (2010). Psicología social. México DF: Cenage Learning.

Kenney, M.G. (20II). An opportunity for higher education: using social entrepreneurship instruction to mitigate social problems. Journal of business studies quarterly, 3(2), 77-83. Recuperado de http://jbsq.org/ wp-content/uploads/20II/I2/Dec_20II_7.pdf

La Jornada. (2015). Crecen comercios en México, 94\% son microempresas: Inegi. Recuperado de http://www.jornada.unam.mx/ultimas/2015/07/28/ aumentan-comercios-en-mexico-el-94-son-microempresas-inegi-9993. html

Limonero, J.T., Tomás-Sábado, J., Fernández-Castro, J., Gómez-Romero, M.J. y Ardilla-Herrero, A. (2012). Estrategias de afrontamiento resilientes y regulación emocional: predictores de satisfacción con la vida. Behavioral psychology, 20(I), I83-I96. Recuperado de https://www.researchgate. net/publication/234139556_Resilient_coping_strategies_and_ emotion_regulation_predictors_of_life_satisfaction_Estrategias_de_ afrontamiento_resilientes_y_regulacion_emocional_predictores_de_ satisfaccion_con_la_vida

Marchesi, Á. (2009). Desarrollo psicológico y educación: 3. Trastornos del desarrollo y necesidades educativas especiales. Madrid: Alianza Editorial.

Mathinsen, M.E., Merino, J., Castro, G., Mora, O. y Navarro, G. (20II). Adaptación socioemocional en escolares: evaluación de un instrumento nuevo en la provincia de Concepción. Estudios Pedagógicos, 37(2), 6I-75. Recuperado de http://www.redalyc.org/articulo.oa?id=I73520953002

Martínez, G.A. y Campos, J.A. (Abril, 20I4). La intención emprendedora en estudiantes universitarios: el caso de la universidad de Deusto. Boletín de estudios económicos, 59(2II), I5I-I72.

Núñez, M.A., Mercado, P. y Madrigal, B.E. (2014). Autoestima y habilidades emprendedoras en estudiantes de ciencias económico-administrativas de la Universidad de Guadalajara-México. Comuni@acción: Revista de investigación en comunicación y desarrollo, 5(2), I3-24. Recuperado de http:// www.redalyc.org/articulo.oa?id $=449844868002$

Osorio, F.F. y Pereira, F. (20II). Hacia un modelo de educación para el emprendimiento: una mirada desde la teoría social cognitiva. En Cuadernos 
de Administración, 24(43), 13-33. Recuperado de http://www.redalyc. org $/$ articulo.oa?id=2052I43500I

Orazem, P.F., Jolly, R. \& Yu, L. (2015). Once an entrepreneur, always an entrepreneur? The impacts of skills developed before, during and after college on firm start-ups. IZA Journal of Labor Economics, 4(9), I-27. Recuperado de http://link.springer.com/article/IO.II86/s40172015-0023-7\#article-actions

Saarni, C. (200Ia). Cognition, Context and Goals: Significant Components in Social-Emotional Effectiveness. Social Development, $10(\mathrm{I})$, I25-I29. Recuperado de http://onlinelibrary.wiley.com/doi/I0.I II I/I4679507.00I52/abstract, doi: IO.I I I I/I467-9507.00I52

Saarni, C. (200Ib). Epilogue: Emotion communication and relationship context. International Journal of Behavioral Development, 25(4), 354-356. Recuperado de http://www.tandf.co.uk/journals/pp/0I650254.html. Doi: I0.1080/0I650250143000094

Santa Rita, I. (2013). Enrique Peña: El impacto a tu bolsillo. Recuperado de http:/ / www.cnnexpansion.com/mi-dinero/2013/I I/25/acciones-de-epnque-impactan-tu-bolsillo

Sánchez, G. y Araya-Vargas, G. (2013). Atención plena, inteligencia emocional, género, área de estudio y reporte de ejercicio en estudiantes universitarios costarricenses. Revista Iberoamericana de Psicología del Ejercicio y el Deporte, 9(I), I9-36. Recuperado de http://www.redalyc.org/articulo. oa?id=3I I I30I990I4

Scafarelli, L.M. y García, R.C. (2010). Estrategias de afrontamiento al estrés en una muestra de jóvenes universitarios uruguayos. Ciencias psicológicas, 4(2), I65-I75. Recuperado de http://www.scielo.edu.uy/pdf/cp/ $\mathrm{v} 4 \mathrm{n} 2 / \mathrm{v} 4 \mathrm{n} 2 \mathrm{aO} 4 . \mathrm{pdf}$ 